DOI 10.4171/JEMS/270

Jacopo Stoppa · Gábor Székelyhidi

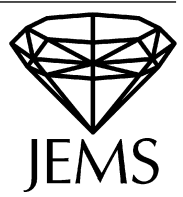

\title{
Relative K-stability of extremal metrics
}

Received January 14, 2010 and in revised form June 9, 2010

Abstract. We show that if a polarised manifold admits an extremal metric then it is K-polystable relative to a maximal torus of automorphisms.

\section{Introduction}

Calabi [4] introduced the notion of extremal metrics as candidates for canonical representatives of Kähler classes on compact Kähler manifolds. Unfortunately not all Kähler manifolds admit extremal metrics (see e.g. Levine [11]) and even if they do, they may not admit them in all Kähler classes (see e.g. Apostolov, Calderbank, Gauduchon, TønnesenFriedman [1]). This makes the question of existence of extremal metrics quite delicate and there is now a vast literature on the topic. We refer to Phong-Sturm [14] for a recent survey and an extensive bibliography.

By definition an extremal metric is a Kähler metric whose scalar curvature has holomorphic gradient vector field. Thus, special cases are constant scalar curvature Kähler (or $\operatorname{cscK}$ ) metrics and Kähler-Einstein metrics. While one can study these metrics in arbitrary Kähler classes, perhaps the most interesting case is when the Kähler class is the first Chern class of an ample line bundle. Indeed, existence of a cscK metric on a manifold $M$ in the Kähler class $c_{1}(L)$ for an ample line bundle $L$ is expected to be closely related to algebro-geometric properties of the polarised manifold $(M, L)$. This is expressed by the following.

Conjecture 1.1 (Yau [20], Tian [19], Donaldson [7]). The manifold $M$ admits a cscK metric in the class $c_{1}(L)$ if and only if the pair $(M, L)$ is $K$-polystable.

The notion of K-polystability will be recalled below. Building on the K-semistability proved by Donaldson [8] and on the work of Arezzo-Pacard [2] on blowing up cscK metrics, the first named author completed the proof of one direction of this conjecture, under the assumption that the automorphism group of $(M, L)$ is discrete.

J. Stoppa: Department of Pure Mathematics and Mathematical Statistics, University of Cambridge, Wilberforce Road, Cambridge CB3 0WB, UK; e-mail: J.Stoppa@dpmms.cam.ac.uk

G. Székelyhidi: Department of Mathematics, Columbia University, 2990 Broadway, New York, NY 10027, USA; e-mail: gabor@math.columbia.edu

Mathematics Subject Classification (2010): Primary 53C55; Secondary 53C25 
Theorem 1.2 ([15, Theorem 1.2]). If $M$ admits a cscK metric in $c_{1}(L)$ and $\operatorname{Aut}(M, L)$ is discrete, then $(M, L)$ is $K$-polystable.

Using a different approach, this was recently extended to manifolds with not necessarily discrete automorphism groups by Mabuchi [12], [13]. The aim of the present paper is to generalise this theorem to the case of extremal metrics. In this case the conjecture analogous to Conjecture 1.1 was formulated by the second named author in [18].

Conjecture 1.3. The manifold $M$ admits an extremal metric in the class $c_{1}(L)$ if and only if the pair $(M, L)$ is K-polystable relative to a maximal torus of automorphisms of $(M, L)$.

By generalising the approach in [15] we obtain the following, which is the main result of this paper.

Theorem 1.4. If $M$ admits an extremal metric in $c_{1}(L)$ then $(M, L)$ is $K$-polystable relative to a maximal torus of automorphisms of $(M, L)$.

In particular the theorem applies when $M$ admits a cscK metric and has continuous automorphisms, proving that $M$ is K-polystable with respect to all test-configurations that commute with a maximal torus of automorphisms, but note that this is a priori a weaker condition than K-polystability (see the next section for the detailed definitions).

Note that by an example in [1] relative K-polystability may not be sufficient to ensure the existence of an extremal metric, so it is likely that Conjectures 1.1 and 1.3 have to be refined.

\section{Relative K-polystability}

In this section we recall the notion of relative K-polystability following [18]. This is a modification of the notion of K-polystability introduced by Donaldson [7].

Suppose that $(V, L)$ is a polarised scheme of dimension $n$, with a $\mathbb{C}^{*}$-action $\alpha$. Let us write $A_{k}$ for the infinitesimal generator of the action of $\alpha$ on $H^{0}\left(V, L^{k}\right)$, and write $d_{k}$ for the dimension of $H^{0}\left(V, L^{k}\right)$. Then $d_{k}$ is a polynomial of degree $n$ and $\operatorname{Tr}\left(A_{k}\right)$ is a polynomial of degree $n+1$ for sufficiently large $k$, so we can write

$$
d_{k}=c_{0} k^{n}+c_{1} k^{n-1}+O\left(k^{n-2}\right), \quad \operatorname{Tr}\left(A_{k}\right)=a_{0} k^{n+1}+a_{1} k^{n}+O\left(k^{n-1}\right) .
$$

Donaldson's Futaki invariant is defined to be

$$
F(\alpha)=\frac{c_{1}}{c_{0}} a_{0}-a_{1} .
$$

Sometimes we will write $F(V, L, \alpha)$ to emphasize the space that $\alpha$ is acting on.

Suppose in addition that we have a $\mathbb{C}^{*}$-action $\beta$ on $(V, L)$ which commutes with $\alpha$, and write $B_{k}$ for the infinitesimal generator of the action on $H^{0}\left(V, L^{k}\right)$. Then $\operatorname{Tr}\left(A_{k} B_{k}\right)$ 
is a polynomial of degree $k+2$ for sufficiently large $k$, and we define the inner product $\langle\alpha, \beta\rangle$ to be the leading coefficient in the expansion

$$
\operatorname{Tr}\left(A_{k} B_{k}\right)-\frac{\operatorname{Tr}\left(A_{k}\right) \operatorname{Tr}\left(B_{k}\right)}{d_{k}}=\langle\alpha, \beta\rangle k^{n+2}+O\left(k^{n+1}\right) .
$$

When $V$ is a smooth manifold, this inner product can also be computed differentialgeometrically. It was originally introduced in this form by Futaki-Mabuchi [9].

To define the relative Futaki invariant, suppose that we have a torus action $T$ on $(V, L)$ commuting with $\alpha$. Let us write $\bar{\alpha}$ for the projection of $\alpha$ orthogonal to $T$, with respect to the inner product we have defined. Then we define the relative Futaki invariant $F_{T}(\alpha)$ by

$$
F_{T}(\alpha)=F(\bar{\alpha}) .
$$

Equivalently if $\beta_{1}, \ldots, \beta_{d}$ is a basis of $\mathbb{C}^{*}$-actions generating the torus $T$, then

$$
F_{T}(\alpha)=F(\alpha)-\sum_{i=1}^{d} \frac{\left\langle\alpha, \beta_{i}\right\rangle}{\left\langle\beta_{i}, \beta_{i}\right\rangle} F\left(\beta_{i}\right) .
$$

It will be convenient for us to extend these definitions to $\mathbb{Q}$-line bundles using the relation

$$
F\left(V, L^{r}, \alpha\right)=r^{n} F(V, L, \alpha),
$$

which the reader can readily verify. It will also be useful to allow rational multiples of $\mathbb{C}^{*}$-actions. For this we use the relation

$$
F(V, L, r \alpha)=r F(V, L, \alpha) .
$$

We next recall the notion of a test-configuration from [7] with the necessary modification for relative stability.

Definition 2.1. A test-configuration for $(X, L)$ consists of a $\mathbb{C}^{*}$-equivariant flat family of schemes $\pi: \mathcal{X} \rightarrow \mathbb{C}$ (where $\mathbb{C}^{*}$ acts on $\mathbb{C}$ by multiplication) and a $\mathbb{C}^{*}$-equivariant, relatively ample $\mathbb{Q}$-line bundle $\mathcal{L}$ over $\mathcal{X}$. We require that the fibres $\left(\mathcal{X}_{t}, \mathcal{L}_{\mid \mathcal{X}_{t}}\right)$ are isomorphic to $(X, L)$ for $t \neq 0$, where $\mathcal{X}_{t}=\pi^{-1}(t)$. The test-configuration is called a product configuration if $\mathcal{X}=X \times \mathbb{C}$.

We say that the test-configuration is compatible with a torus $T$ of automorphisms of $(X, L)$ if there is a torus action on $(\mathcal{X}, \mathcal{L})$ which preserves the fibres of $\pi: \mathcal{X} \rightarrow \mathbb{C}$, commutes with the $\mathbb{C}^{*}$-action, and restricts to $T$ on $\left(\mathcal{X}_{t}, \mathcal{L}_{\mid \mathcal{X}_{t}}\right)$ for $t \neq 0$.

Note that given a test-configuration $(\mathcal{X}, \mathcal{L})$, there is an induced $\mathbb{C}^{*}$-action $\alpha$ on the central fibre $\left(\mathcal{X}_{0}, \mathcal{L}_{\mid \mathcal{X}_{0}}\right)$. We will write $F(\mathcal{X}, \mathcal{L})$ for the Futaki invariant of this induced action $\alpha$. With these preliminaries we can state the main definition.

Definition 2.2. A polarised variety $(X, L)$ is $K$-semistable relative to a torus $T$ of automorphisms if $F_{T}(\mathcal{X}, \mathcal{L}) \geq 0$ for all test-configurations compatible with the torus. If in addition equality holds only for the product configuration, then $(X, L)$ is $K$-polystable relative to the torus $T$. 
If we have two tori $T^{\prime} \subset T$ acting on $(X, L)$, then $\mathrm{K}$-polystability relative to $T$ is a weaker condition than relative to $T^{\prime}$, since there are fewer test-configurations compatible with a larger torus. Thus, the weakest notion is $\mathrm{K}$-polystability relative to a maximal torus of automorphisms. The strongest notion is K-polystability relative to the extremal $\mathbb{C}^{*}$ action. This is a $\mathbb{C}^{*}$-action $\chi$ defined by Futaki-Mabuchi [9] as follows. Fix a maximal torus of automorphisms $T$, and write $\mathfrak{t}$ for its Lie algebra. The Futaki invariant gives a linear map $\mathfrak{t} \mapsto \mathbb{C}$, and $\chi$ is dual to this map under the inner product on $\mathfrak{t}$. This gives a $\mathbb{C}^{*}$-action on $(X, L)$, unique up to conjugation. In particular if the Futaki invariant of any $\mathbb{C}^{*}$-action on $(X, L)$ vanishes, then $\chi=0$, and $\mathrm{K}$-polystability relative to $\chi$ is simply K-polystability.

It would be interesting to strengthen the conclusion of Theorem 1.4 to K-polystability relative to the extremal $\mathbb{C}^{*}$-action. Note that the analogous statement is true in finite dimensional geometric invariant theory, by Theorem 3.5 in [18] (the same proof works if we replace the maximal torus with any torus containing the extremal $\mathbb{C}^{*}$-action).

We next recall the two theorems that we will use in the next section.

Theorem 2.3. If $M$ admits an extremal metric in $c_{1}(L)$ then $(M, L)$ is $K$-semistable relative to a maximal torus of automorphisms.

Proof. This follows easily from Donaldson's lower bound for the Calabi functional [8]. For details see [17]. For the convenience of the reader we outline the argument here. Donaldson's lower bound tells us that for any test-configuration, if $\alpha$ is the induced $\mathbb{C}^{*}$ action on the central fibre, then

$$
\inf _{\omega \in c_{1}(L)} c_{n}\|S(\omega)-\hat{S}\|_{L^{2}} \geq \frac{-F(\alpha)}{\|\alpha\|},
$$

where $c_{n}$ is a constant depending only on the dimension, $\|\alpha\|=\langle\alpha, \alpha\rangle^{1 / 2}$ using the inner product defined above, and $\hat{S}$ is the average of the scalar curvature $S(\omega)$. Moreover, if $\omega$ is an extremal metric, then

$$
c_{n}\|S(\omega)-\hat{S}\|_{L^{2}}=\frac{F(\chi)}{\|\chi\|}=\|\chi\|,
$$

where $\chi$ is the extremal vector field on $(M, L)$. We are using here the fact that $F(\chi)=$ $\langle\chi, \chi\rangle$ by definition of the extremal vector field. It follows from 2.1) and 2.2) that if $M$ admits an extremal metric in $c_{1}(L)$ then

$$
\frac{F(\alpha)}{\|\alpha\|} \geq-\|\chi\|
$$

for all test-configurations.

Suppose now that $M$ admits an extremal metric in $c_{1}(L)$, and we have a test-configuration for $(M, L)$ which is compatible with a maximal torus of automorphisms $T$. Write $\alpha$ for the induced $\mathbb{C}^{*}$-action on the central fibre. By twisting the $\mathbb{C}^{*}$-action on the total space by the projection of $\alpha$ onto $T$ if necessary, we can assume that $\alpha$ is orthogonal to $T$. We want to show that $F(\alpha) \geq 0$. Suppose on the contrary that $F(\alpha)<0$, and let 
$\mu>0$ satisfy $F(\mu \alpha)=-\|\mu \alpha\|^{2}$. By pulling back the test-configuration under a base change $z \mapsto z^{r}$, and twisting the action on the total space by the inverse of $\chi$, we obtain a test-configuration for $(M, L)$ such that the action on the central fibre is $r(\mu \alpha-\chi)$, where $r$ is large enough to make this a genuine $\mathbb{C}^{*}$-action. From 2.3 we know that

$$
\frac{F(\mu \alpha-\chi)}{\|\mu \alpha-\chi\|}=\frac{F(r(\mu \alpha-\chi))}{\|r(\mu \alpha-\chi)\|} \geq-\|\chi\| .
$$

But at the same time

$$
F(\mu \alpha-\chi)=-\|\mu \alpha\|^{2}-\|\chi\|^{2}=-\|\mu \alpha-\chi\|^{2},
$$

since $\alpha$ is orthogonal to $\chi$. So

$$
\frac{F(\mu \alpha-\chi)}{\|\mu \alpha-\chi\|}=-\|\mu \alpha-\chi\|<-\|\chi\| .
$$

This contradiction shows that $(M, L)$ is $\mathrm{K}$-polystable relative to $T$. The same argument also shows that $(M, L)$ is $\mathrm{K}$-polystable relative to the extremal $\mathbb{C}^{*}$-action.

Theorem 2.4 (Arezzo-Pacard-Singer [3]). Suppose that $M$ admits an extremal metric in $c_{1}(L)$, and let $T$ be a maximal torus of automorphisms of $(M, L)$. If $p \in M$ is a fixed point of $T$, then the blowup $\mathrm{Bl}_{p} M$ of $M$ at $p$ admits an extremal metric in the class $c_{1}\left(\pi^{*} L-\varepsilon E\right)$ for sufficiently small $\varepsilon>0$. Here $\pi$ is the blowdown map, and $E$ is the exceptional divisor.

Proof. This follows from [3, Theorem 2.1]. Indeed we can choose an extremal metric $\omega$ on $M$ such that the isometry group of $\omega$ contains a compact maximal torus $T_{\mathbb{R}}$, which is contained in the complex torus $T$. In the notation of [3] we let $K=T_{\mathbb{R}}$, and let $\mathfrak{k}$ be its Lie algebra. Since $K$ is a maximal torus, any $K$-invariant holomorphic hamiltonian vector field lies in $\mathfrak{k}$. Moreover if we write $S(\omega)$ for the scalar curvature then by Calabi's theorem [5] the vector field $J \nabla S(\omega)$ lies in the centre of the Lie algebra of Killing fields, so it also lies in $\mathfrak{k}$. This allows us to apply [3, Theorem 2.1], and we get the stated result.

\section{Proof of Theorem 1.4}

Let us suppose that $M$ admits an extremal metric in $c_{1}(L)$ and choose a maximal torus $T \subset \operatorname{Aut}(M, L)$. From Theorem 2.3 we know that if $(\mathcal{X}, \mathcal{L})$ is a test-configuration for $(M, L)$ compatible with $T$, then the relative Futaki invariant satisfies $F_{T}(\mathcal{X}) \geq 0$. Suppose then that $F_{T}(\mathcal{X})=0$.

We can assume that $M \subset \mathbb{P}(V)$, where $V=H^{0}(M, L)^{*}$. Moreover the torus $T$ acts on $\mathbb{P}(V)$, preserving $M$. In addition there is an extra $\mathbb{C}^{*}$-action $\alpha$ on $\mathbb{P}(V)$, commuting with the $T$-action and such that the flat closure of the family $t \mapsto \alpha(t) \cdot M$ across $t=0$ is the test-configuration $\mathcal{X}$. Let us write $\left(M_{0}, L_{0}\right)$ for the central fibre of the testconfiguration. Then we have both $\alpha$ and the torus $T$ acting on $\left(M_{0}, L_{0}\right)$. By twisting the 
action on the total space by the orthogonal projection of $\alpha$ onto $T$ (which does not change the relative Futaki invariant), we can assume that $\langle\alpha, T\rangle=0$. In this case

$$
F_{T}(\mathcal{X}, \mathcal{L})=F\left(M_{0}, L_{0}, \alpha\right) .
$$

We now look at the weight decomposition under $\alpha$ given by

$$
V=\bigoplus_{i} V_{m_{i}},
$$

where $m_{0}<m_{1}<\cdots<m_{L}$ for some $L>0$, and consider the least $l \geq 0$ such that

$$
\operatorname{red}\left(M_{0}\right) \subset \mathbb{P}\left(\bigoplus_{i \leq l} V_{m_{i}}\right) .
$$

It is proved in [15, Section 3] that if $l=0$, so that $\alpha$ acts trivially on $\operatorname{red}\left(M_{0}\right)$, then either $\mathcal{X}$ is a product test-configuration, or $F\left(M_{0}, L_{0}, \alpha\right)>0$, which is a contradiction.

Remark. Following an observation of S. Donaldson, we may alternatively take for $V$ a suitable subspace of $H^{0}\left(M, L^{p}\right)^{*}$ (invariant under $\alpha$ and $T$ ), for $p \gg 1$, i.e. take a different embedding of the same test configuration given by a Veronese embedding followed by a linear projection. One advantage is that in this way we can assume that $M_{0}$ does not lie on a proper projective subspace of $\mathbb{P}(V)$. Thus in the $l=0$ case above we would get immediately $M_{0} \neq \operatorname{red}\left(M_{0}\right)$, which is an important step in [15, Section 3].

On the other hand, if $l>0$, then consider the repulsive fixed point set

$$
M_{0}^{\prime}=\operatorname{red}\left(M_{0}\right) \cap \mathbb{P}\left(V_{m_{l}}\right) .
$$

The set of points $p \in M$ for which the limit

$$
q=\lim _{t \rightarrow 0} \alpha(t) p
$$

is in $M_{0}^{\prime}$ is precisely

$$
M^{\prime}=M \cap \mathbb{P}\left(\bigoplus_{i \geq l} V_{m_{i}}\right) .
$$

This is a closed $T$-invariant set, so it contains a point $p$ fixed by $T$. To see this, we can take a basis of $\mathbb{C}^{*}$-actions $\beta_{i}$ generating the torus $T$, and then given any point $p$ in $M^{\prime}$ we can inductively move it to a fixed point of $\beta_{i}$ by taking the limit of $\beta_{i}(t) p$ as $t \rightarrow 0$. Doing this for each $i$, we end up with a fixed point of $T$. The corresponding limit $q$ will then be a $T$-invariant, repulsive fixed point of $\alpha$ in $\operatorname{red}\left(M_{0}\right)$.

Letting $Z \subset \mathcal{X}$ be the closure of the orbit of $p$ under $\alpha$, we obtain a test-configuration

$$
(\widehat{\mathcal{X}}, \widehat{\mathcal{L}})=\left(\mathrm{Bl}_{Z} \mathcal{X}, \phi^{*} \mathcal{L}-\varepsilon E\right)
$$

for the polarised manifold $\left(\mathrm{Bl}_{p} M, \phi^{*} L-\varepsilon E\right)$, where $\phi: \widehat{\mathcal{X}} \rightarrow \mathcal{X}$ is the blowdown. The only nontrivial thing to check is flatness of the composition $\pi \circ \phi: \widehat{\mathcal{X}} \rightarrow \mathcal{X} \rightarrow \mathbb{C}$. This holds because blowing up $Z \subset \mathcal{X}$ does not introduce new associated points (i.e. 
embedded schemes) of $\mathcal{X}$, only the Cartier exceptional divisor $E$ (for details see the proof of Proposition 2.13 of [15]).

For suitably small $\varepsilon>0$ the test-configuration $(\widehat{\mathcal{X}}, \widehat{\mathcal{L}})$ will have negative Futaki invariant, and in fact it will even have negative Futaki invariant relative to $T$. This follows from the lemma below and its corollary.

At the same time from Theorem 2.4 we know that $\mathrm{Bl}_{p} M$ admits an extremal metric in the class $c_{1}\left(\phi^{*} L-\varepsilon E\right)$ for suitably small $\varepsilon$ since $p$ is fixed by the torus $T$, which is a maximal torus of automorphisms of $M$. This contradicts Theorem 2.3. and completes the proof of the main theorem.

Lemma 3.1. Let $(\mathcal{X}, \mathcal{L})$ be a test-configuration for $(M, L)$ compatible with a torus $T$ of automorphisms, and suppose that the induced action $\alpha$ on the central fibre satisfies $\langle\alpha, T\rangle=0$. Let $\widehat{\mathcal{X}}$ be given by the blowup of a $T$-invariant section as described above. Then

$$
F(\widehat{\mathcal{X}}, \widehat{\mathcal{L}})=F(\mathcal{X}, \mathcal{L})+\left(\lambda(q)-\frac{b_{0}}{a_{0}}\right) \frac{\varepsilon^{n-1}}{2(n-2) !}+O\left(\varepsilon^{n}\right),
$$

and

$$
\langle\hat{\alpha}, \hat{T}\rangle=O\left(\varepsilon^{n}\right),
$$

where we use the $\mathbb{Q}$-polarization $\widehat{\mathcal{L}}=\phi^{*} \mathcal{L}-\varepsilon E$ on $\widehat{\mathcal{X}}$ for some small rational $\varepsilon>0$, and $\hat{\alpha}, \hat{T}$ are the actions of $\alpha$ and $T$ lifted to the blowup. It follows that the relative Futaki invariants satisfy

$$
F_{T}(\widehat{\mathcal{X}}, \widehat{\mathcal{L}})=F_{T}(\mathcal{X}, \mathcal{L})+\left(\lambda(q)-\frac{b_{0}}{a_{0}}\right) \frac{\varepsilon^{n-1}}{2(n-2) !}+O\left(\varepsilon^{n}\right) .
$$

Here $\lambda(q)$ is the weight of $\alpha$ on the fibre $L_{0 \mid q}$, and $a_{0}, b_{0}$ are defined by the expansions of the dimension and weight on $H^{0}\left(M_{0}, L_{0}^{k}\right)$ calculated at the central fibre of $\mathcal{X}$ as usual:

$$
d_{k}=a_{0} k^{n}+a_{1} k^{n-1}+\cdots, \quad w_{k}=b_{0} k^{n+1}+b_{1} k^{n}+\cdots .
$$

Proof. The central fibre of $\widehat{\mathcal{X}}$ will not in general be isomorphic to $\widehat{M}_{0}:=\mathrm{Bl}_{q} M_{0}$. In fact it will contain another large component $P$ glued to $\widehat{M}_{0}$ along the exceptional divisor $E^{\prime}$ for the morphism $\widehat{M}_{0} \rightarrow M_{0}$, as we now explain.

By [10, II Corollary 7.15], there is a closed immersion $\widehat{M}_{0} \hookrightarrow \widehat{\mathcal{X}}_{0}$ induced by the closed immersion $M_{0} \subset \mathcal{X}$ under blowing up $Z$. Let $\mathcal{I}_{q} \subset \mathcal{O}_{M_{0}}$ denote the ideal sheaf of $q \in M_{0}$. By the algebraic definition of blowing up we have $\widehat{M}_{0} \cong \operatorname{Proj} \bigoplus_{k \geq 0} \mathcal{I}_{q}^{k}$. On the other hand the generic fibre of $\widehat{\mathcal{X}}$ is $\operatorname{Proj} \bigoplus_{k>0} \mathcal{I}_{p}^{k}$, where $\mathcal{I}_{p}$ is the ideal of the smooth point $p \in M$. Thus by the numerical criterion for flatness when the Hilbert-Samuel polynomial for $p \in M$ is larger than that of $q \in M_{0}$ (i.e. when $q$ is singular enough as a point of $M_{0}$ ) there will be an additional component $P$ in the central fibre, given by the closure of $\widehat{\mathcal{X}}_{0} \backslash \widehat{M}_{0}$. A simple example has been suggested by S. Donaldson: when $q$ is an isolated threefold ordinary double point inside the central fibre one has $P \cong \mathbb{P}^{3}$ glued in along a smooth quadric. Note that this is different from the situation described in [16, Section 2], where the central fibre of the original test-configuration is smooth (isomorphic 
to $M$ ), but one blows up 0 -cycles instead of just a point. In any case the restriction $\widehat{\mathcal{L}}_{0 \mid} \widehat{M}_{0}$ is just $\phi^{*} L_{0}-\varepsilon E^{\prime}$ (recall we defined $E^{\prime}$ as the exceptional divisor for blowing up $q$ on the central fibre).

Taking this information into account we now compute the Donaldson-Futaki invariant for the action $\alpha$ on the central fibre $\widehat{\mathcal{X}}_{0}$. In the calculations that follow $\varepsilon$ is a fixed positive rational number, and we tacitly restrict to those $k \gg 1$ for which $k \varepsilon$ is an integer. We also suppress pullbacks like $\pi^{*}$ or $\phi^{*}$ when this causes no confusion. By flatness, using the Riemann-Roch theorem we have

$$
\begin{aligned}
h^{0}\left(\widehat{\mathcal{X}}_{0}, \widehat{\mathcal{L}}_{0}^{k}\right) & =h^{0}\left(\mathrm{Bl}_{p} M, L^{k}-k \varepsilon E\right) \\
& =h^{0}\left(M, L^{k}\right)-\frac{\varepsilon^{n}}{n !} k^{n}-\frac{\varepsilon^{n-1}}{2(n-2) !} k^{n-1}+\cdots .
\end{aligned}
$$

Using the restriction $\mathbb{C}^{*}$-equivariant exact sequence

$$
0 \rightarrow H_{P}^{0}\left(\mathcal{I}_{E^{\prime}}^{k \varepsilon} \widehat{\mathcal{L}}_{0 \mid P}^{k}\right) \rightarrow H_{\widehat{\mathcal{X}}_{0}}^{0}\left(\widehat{\mathcal{L}}_{0}^{k}\right) \rightarrow H_{\widehat{M}_{0}}^{0}\left(L_{0}^{k}-k \varepsilon E^{\prime}\right) \rightarrow 0
$$

which holds for large $k \gg 1$, we find

$$
\operatorname{Tr}\left(H_{\widehat{\mathcal{X}}_{0}}^{0}\left(\widehat{\mathcal{L}}_{0}^{k}\right)\right)=\operatorname{Tr}\left(H_{\widehat{M}_{0}}^{0}\left(L_{0}^{k}-k \varepsilon E^{\prime}\right)\right)+\operatorname{Tr}\left(H_{P}^{0}\left(\mathcal{I}_{E^{\prime}}^{r} \widehat{\mathcal{L}}_{0 \mid p}^{k}\right)\right) .
$$

Note that $H_{\widehat{M}_{0}}^{0}\left(L_{0}^{k}-k \varepsilon E^{\prime}\right) \cong H_{M_{0}}^{0}\left(\mathcal{I}_{q}^{k \varepsilon} L_{0}^{k}\right)$ so the first term in the formula above equals $\operatorname{Tr}\left(H_{M_{0}}^{0}\left(L_{0}^{k}\right)\right)-\operatorname{Tr}\left(H^{0}\left(\mathcal{O}_{k \varepsilon q} \otimes L_{\left.0\right|_{q}}^{k}\right)\right)$. From the exact sequence

$$
0 \rightarrow \mathcal{I}_{q}^{k \varepsilon} L_{0}^{k} \rightarrow L_{0}^{k} \rightarrow \mathcal{O}_{k \varepsilon q} \otimes L_{\left.0\right|_{q}}^{k} \rightarrow 0,
$$

together with 3.2 and 3.3 we see that the length of the $\mathcal{O}_{M_{0}}$-module $\mathcal{O}_{k \varepsilon q}$ is given by

$$
h_{P}^{0}\left(\mathcal{I}_{E^{\prime}}^{k \varepsilon} \widehat{\mathcal{L}}_{0 \mid P}^{k}\right)+\frac{\varepsilon^{n}}{n !} k^{n}+\frac{\varepsilon^{n-1}}{2(n-2) !} k^{n-1}+O\left(k^{n-2}\right) .
$$

It follows that the weight of the action on $\left.\mathcal{O}_{k \varepsilon q} \otimes L^{k}\right|_{q}$ is given by

$$
\begin{aligned}
w\left(\mathcal{O}_{k \varepsilon q} \otimes L_{\left.\right|_{q}}^{k}\right)= & w\left(\mathcal{O}_{k \varepsilon q}\right)+k \lambda(q) \operatorname{len}\left(\mathcal{O}_{k \varepsilon q}\right) \\
= & k \lambda(q) h_{P}^{0}\left(\mathcal{I}_{E^{\prime}}^{k \varepsilon} \widehat{\mathcal{L}}_{0 \mid P}^{k}\right) \\
& +\left(c_{0} \varepsilon^{n+1}+\lambda(q) \frac{\varepsilon^{n}}{n !}\right) k^{n+1}+\left(c_{1} \varepsilon^{n}+\lambda(q) \frac{\varepsilon^{n-1}}{2(n-2) !}\right) k^{n}+\cdots,
\end{aligned}
$$

where $c_{0}, c_{1}$ are given by the expansion

$$
w\left(\mathcal{O}_{k \varepsilon q}\right)=c_{0}(k \varepsilon)^{n+1}+c_{1}(k \varepsilon)^{n}+\cdots .
$$

Similarly $\mathcal{I}_{E^{\prime}}^{k \varepsilon} \widehat{\mathcal{L}}_{\left.0\right|_{P}}^{k} \cong \mathcal{L}_{\left.0\right|_{q}}^{k} \otimes \mathcal{I}_{E^{\prime}}^{k \varepsilon} \mathcal{O}(-k \varepsilon E)_{\left.\right|_{P}}$, so one has

$$
\operatorname{Tr}\left(H_{P}^{0}\left(\mathcal{I}_{E^{\prime}}^{k \varepsilon} \widehat{\mathcal{L}}_{0 \mid P}^{k}\right)\right)=k \lambda(q) h_{P}^{0}\left(\mathcal{I}_{E^{\prime}}^{k \varepsilon} \widehat{\mathcal{L}}_{0 \mid P}^{k}\right)+\cdots
$$


up to a polynomial in $k \varepsilon$ of degree $n+1$. After a simple cancellation we find

$$
\begin{array}{ll}
\hat{a}_{0}=a_{0}+O\left(\varepsilon^{n}\right), & \hat{b}_{0}=b_{0}+O\left(\varepsilon^{n}\right), \\
\hat{a}_{1}=a_{1}-\frac{\varepsilon^{n-1}}{2(n-2) !}, & \hat{b}_{1}=b_{1}-\lambda(q) \frac{\varepsilon^{n-1}}{2(n-2) !},
\end{array}
$$

where $\hat{a}_{i}, \hat{b}_{i}$ are computed on $\widehat{\mathcal{X}}$. Using the formula $F(\widehat{\mathcal{X}})=\left(\hat{a}_{1} / \hat{a}_{0}\right) \hat{b}_{0}-\hat{b}_{1}$ we get

$$
F(\widehat{\mathcal{X}})=F(\mathcal{X})+\left(\lambda(q)-\frac{b_{0}}{a_{0}}\right) \frac{\varepsilon^{n-1}}{2(n-2) !}+O\left(\varepsilon^{n}\right) .
$$

Now let $\beta$ be any $\mathbb{C}^{*}$-action in the torus $T$. To compute the inner product $\langle\hat{\alpha}, \hat{\beta}\rangle$, let us write $A_{k}, B_{k}$ for the infinitesimal generators of the actions $\alpha, \beta$ on $H^{0}\left(M_{0}, L_{0}^{k}\right)$, and $\hat{A}_{k}, \hat{B}_{k}$ for the infinitesimal actions of the corresponding actions on $H^{0}\left(\widehat{\mathcal{X}}_{0}, \widehat{\mathcal{L}}_{0}^{k}\right)$. The inner product $\langle\hat{\alpha}, \hat{\beta}\rangle$ is the leading order term in

$$
\operatorname{Tr}\left(\hat{A}_{k} \hat{B}_{k}\right)-\frac{\operatorname{Tr}\left(\hat{A}_{k}\right) \operatorname{Tr}\left(\hat{B}_{k}\right)}{\hat{d}_{k}} .
$$

Since the actions $\alpha, \beta$ commute, we can use precisely the same exact sequences as before to compute

$$
\begin{aligned}
\operatorname{Tr}\left(A_{k} B_{k}\right)-\operatorname{Tr}\left(\hat{A}_{k} \hat{B}_{k}\right) & =\lambda_{\alpha}(p) \lambda_{\beta}(p)\left(\operatorname{len}\left(\mathcal{O}_{k \varepsilon p}\right)-h_{P}^{0}\left(\mathcal{I}_{E^{\prime}}^{k \varepsilon} \widehat{\mathcal{L}}_{0 \mid P}^{k}\right)\right)+d_{0} k^{n+2} \\
& +\operatorname{Tr}\left(A_{k \varepsilon}^{\prime} B_{k \varepsilon}^{\prime}\right),
\end{aligned}
$$

where $A_{k \varepsilon}^{\prime}$ and $B_{k \varepsilon}^{\prime}$ are the infinitesimal generators of the actions $\alpha, \beta$ on $\mathcal{O}_{k \varepsilon p}$. We have an expansion

$$
\operatorname{Tr}\left(A_{k \varepsilon}^{\prime} B_{k \varepsilon}^{\prime}\right)=c_{0}^{\prime}(\varepsilon k)^{n+2}+O\left(k^{n+1}\right) .
$$

So up to terms of order $\varepsilon^{n}$, the leading order term in (3.4) is the same as that in

$$
\operatorname{Tr}\left(A_{k} B_{k}\right)-\frac{\operatorname{Tr}\left(A_{k}\right) \operatorname{Tr}\left(B_{k}\right)}{d_{k}}
$$

which is just $\langle\alpha, \beta\rangle=0$. This shows $\langle\hat{\alpha}, \hat{\beta}\rangle=O\left(\varepsilon^{n}\right)$. A similar computation of the inner product on the blowup is in [6].

The statement about the relative Futaki invariants now follows from the definition

$$
F_{T}(\widehat{\mathcal{X}}, \hat{\alpha})=F(\widehat{\mathcal{X}}, \hat{\alpha})-\sum_{i=1}^{d} \frac{\left\langle\hat{\alpha}, \hat{\beta}_{i}\right\rangle}{\left\langle\hat{\beta}_{i}, \hat{\beta}_{i}\right\rangle} F\left(\widehat{\mathcal{X}}, \hat{\beta}_{i}\right),
$$

where the $\mathbb{C}^{*}$-actions $\beta_{i}$ generate the torus $T$.

Corollary 3.2. In the notation above, if $q \in M_{0}$ is a repulsive fixed point for $\alpha$ then $F(\widehat{\mathcal{X}})<F(\mathcal{X})$ for $\varepsilon$ small enough. 
Proof. It remains to prove that the highest order correction term

$$
\left(\lambda(q)-\frac{b_{0}}{a_{0}}\right) \frac{\varepsilon^{n-1}}{2(n-2) !}
$$

is negative. It is proved is [16, Section 4] that, possibly after a fixed base change of the test-configuration, the coefficient $\lambda(q)-b_{0} / a_{0}$ is integral and equals minus the HilbertMumford weight of $q$ under the induced action of $\alpha$ on $\mathbb{P}(V)$. The Hilbert-Mumford criterion combined with a local computation then shows that the weight of such a repulsive fixed point must be positive (for details see the proof of Theorem 1.2 in [15]).

Alternatively we can give a self-contained proof as follows. Let $M_{0}$ be the central fibre of our test-configuration and suppose that $q$ is a repulsive fixed point with weight $m_{l}$ and also let $r$ be a point in $\operatorname{red}\left(M_{0}\right) \cap \mathbb{P}\left(V_{m_{0}}\right)$, i.e. a lowest weight invariant point. Then as in the Futaki invariant calculation we have the exact sequence

$$
0 \rightarrow \mathcal{I}_{r}^{k \varepsilon} L_{0}^{k} \rightarrow L_{0}^{k} \rightarrow \mathcal{O}_{k \varepsilon r} \otimes L_{\left.0\right|_{r}}^{k} \rightarrow 0
$$

Write $-\lambda$ for the weight $m_{l}$, so $\lambda(q)=\lambda$ and $m_{0} \leq-\lambda-1$. The weights on $L_{0}$ are the opposite by duality and they are all at least $\lambda$. Using the notation from the proof of Theorem 1.4, from the exact sequence we have

$$
\begin{aligned}
w_{k} & =w\left(\mathcal{I}_{r}^{k \varepsilon} L^{k}\right)+w\left(\mathcal{O}_{k \varepsilon r}\right)-k m_{0} \operatorname{len}\left(\mathcal{O}_{k \varepsilon r}\right) \\
& \geq k \lambda\left(d_{k}-\operatorname{len}\left(\mathcal{O}_{k \varepsilon r}\right)\right)+w\left(\mathcal{O}_{k \varepsilon r}\right)+k(\lambda+1) \operatorname{len}\left(\mathcal{O}_{k \varepsilon r}\right) \\
& =k \lambda d_{k}+k \operatorname{len}\left(\mathcal{O}_{k \varepsilon r}\right)+w\left(\mathcal{O}_{k \varepsilon r}\right) .
\end{aligned}
$$

Now we need the expansions

$$
\begin{aligned}
\operatorname{len}\left(\mathcal{O}_{k \varepsilon r}\right) & =c(k \varepsilon)^{n}+O\left(k^{n-1}\right), \\
w\left(\mathcal{O}_{k \varepsilon r}\right) & =c^{\prime}(k \varepsilon)^{n+1}+O\left(k^{n}\right) .
\end{aligned}
$$

It is important here that $c>0$. This follows from [10, III Corollary 9.6]. Then looking at the $k^{n+1}$ term in 3.5 we get

$$
b_{0} \geq \lambda a_{0}+c \varepsilon^{n}+c^{\prime} \varepsilon^{n+1} .
$$

When $\varepsilon$ is chosen sufficiently small we get the required inequality $b_{0} / a_{0}>\lambda$.

Acknowledgments. We would like to thank Julius Ross and Richard Thomas for helpful discussions, and an anonymous referee for useful suggestions. The second named author would also like to thank D. H. Phong for his encouragement and support. This research was partially supported by Trinity College, Cambridge and by NSF grant DMS-0904223. 


\section{References}

[1] Apostolov, V., Calderbank, D. M. J., Gauduchon, P., Tønnesen-Friedman, C. W.: Hamiltonian 2-forms in Kähler geometry III, extremal metrics and stability. Invent. Math. 173, 547-601 (2008) Zbl 1145.53055 MR 2425136

[2] Arezzo, C., Pacard, F.: Blowing up and desingularizing constant scalar curvature Kähler manifolds. Acta Math. 196, 179-228 (2006) Zbl 1123.53036 MR 2275832

[3] Arezzo, C., Pacard, F., Singer, M.: Extremal metrics on blow ups. arXiv:math/0701028

[4] Calabi, E.: Extremal Kähler metrics. In: Seminar on Differential Geometry, S. T. Yau (ed.), Princeton Univ. Press, 259-290 (1982) Zbl 0487.53057 MR 0645743

[5] Calabi, E.: Extremal Kähler metrics II. In: Differential Geometry and Complex Analysis, I. Chavel and H. M. Farkas (eds.), Springer, 95-114 (1985) Zbl 0574.58006 MR 0780039

[6] Della Vedova, A.: CM-stability of blow-ups and canonical metrics. arXiv:0810.5584

[7] Donaldson, S. K.: Scalar curvature and stability of toric varieties. J. Differential Geom. 62, 289-349 (2002) Zbl 1074.53059 MR 1988506

[8] Donaldson, S. K.: Lower bounds on the Calabi functional. J. Differential Geom. 70, 453-472 (2005) Zbl 1149.53042 MR 2192937

[9] Futaki, A., Mabuchi, T.: Bilinear forms and extremal Kähler vector fields associated with Kähler classes. Math. Ann. 301, 199-210 (1995) Zbl 0831.53042 MR 1314584

[10] Hartshorne, R.: Algebraic Geometry. Springer, Heidelberg (1977) Zbl 0367.14001 MR 0463157

[11] Levine, M.: A remark on extremal Kähler metrics. J. Differential Geom. 21, 73-77 (1985) Zbl 0601.53056 MR 0806703

[12] Mabuchi, T.: K-stability of constant scalar curvature polarization. arXiv:0812.4093

[13] Mabuchi, T.: A stronger concept of K-stability. arXiv:0910.4617

[14] Phong, D. H., Sturm, J.: Lectures on stability and constant scalar curvature. In: Current Developments in Mathematics, 2007, Int. Press, Somerville, MA, 101-176 (2009) Zbl 1188.53081 MR 2532997

[15] Stoppa, J.: K-stability of constant scalar curvature Kähler manifolds. Adv. Math. 221, 13971408 (2009) Zbl 1181.53060 MR 2518643

[16] Stoppa, J.: Unstable blowups. J. Algebraic Geom. 19, 1-17 (2010) Zbl 1198.14046 MR 2551756

[17] Székelyhidi, G.: Extremal metrics and $K$-stability. Ph.D. thesis, Imperial College, London (2006)

[18] Székelyhidi, G.: Extremal metrics and $K$-stability. Bull. London Math. Soc. 39, 76-84 (2007) Zbl 1111.53057 MR 2303522

[19] Tian, G.: Kähler-Einstein metrics with positive scalar curvature. Invent. Math. 137, 1-37 (1997) Zbl 0892.53027 MR 1471884

[20] Yau, S.-T.: Open problems in geometry. In: Proc. Sympos. Pure Math. 54, Amer. Math. Soc., 1-28 (1993) Zbl 0801.53001 MR 1216573 